\title{
Frontalis Sling Using a Silicone Rod for Ptosis in Third Nerve Palsy: Cosmesis versus Safety
}

\author{
Yong Min Choi, Namju Kim \\ Department of Ophthalmology, Seoul National University Bundang Hospital, Seongnam, Korea
}

\begin{abstract}
Purpose: To evaluate the results of the frontalis sling operation using a silicone rod for the correction of ptosis in patients with third nerve palsy with a focus on corneal safety.

Methods: Patients with third nerve palsy who underwent the frontalis sling operation using a silicone rod between 2008 and 2019 were included in this study. The medical records of all patients were reviewed, and their clinical characteristics and postoperative outcomes were analyzed. In this retrospective, interventional case series, the main outcome measures were eyelid contour, eyelid height by margin reflex distance, and corneal status.

Results: Twenty-four eyes of 18 patients (12 male and six female patients) were included. The mean age at the time of surgery was 35.1 years (range, 5-64 years). Twelve patients underwent a unilateral ptosis operation, and six patients received a bilateral ptosis operation. The mean follow-up period was 32.1 months (range, 2-87 months). Most patients (21 of 24 eyes, $88 \%)$ showed poor Bell's phenomenon on preoperative examination. Satisfactory eyelid height and eyelid contour were achieved in almost all patients (mean postoperative margin reflex distance, $+1.2 \mathrm{~mm}$ ) postoperatively. Although corneal erosions were detected for several months in eight of 24 eyes after surgery, these findings were well controlled medically with artificial tear eye drops and ointments.

Conclusions: Frontalis sling surgery using a silicone rod can safely and effectively correct ptosis without severe corneal complications in patients with third nerve palsy. Our study outlines a new method to define the postoperative safety outcome by specifically focusing on categorized corneal status.
\end{abstract}

Key Words: Exposure keratopathy, Frontalis sling, Silicone rod, Oculomotor nerve diseases

Third cranial nerve palsy (3rdNP, oculomotor palsy) is a condition caused by damage to the oculomotor nerve, leading to decreased function of the various muscles it controls.

Received: September 9, 2021 Final revision: December 7, 2021 Accepted: December 13, 2021

Corresponding Author: Namju Kim, MD, PhD. Department of Ophthalmology, Seoul National University Bundang Hospital, 82 Gumi-ro, 173beon-gil, Bundang-gu, Seongnam 13620, Korea. Tel: 82-31-787-7376, Fax: 82-31-787-4057, E-mail: kimnamju@snubh.org
This condition occurs due to vascular disease, trauma, aneurysm, or tumors [1]. Common clinical manifestations of 3rdNP are strabismus, limitation of extraocular movement, ptosis, and mydriasis. Blepharoptosis is a common consequence of 3rdNP and can be difficult to manage [2]. The ptotic eyelid may obscure the pupil, causing amblyopia in children and functional and cosmetic problems in adults. The severe ptosis associated with 3rdNP can present challenging management problems. This form of ptosis often involves poor levator muscle function, necessitating 
frontalis sling operation in many cases [3]. However, because of the limited upgaze in patients with 3rdNP, many show poor Bell's phenomenon, making the cornea vulnerable to exposure keratopathy after the surgery. Among the many suspension materials currently used for frontalis sling operations, silicone rods offer good elasticity for favorable eyelid blinking and simple adjustability in revision surgery $[4,5]$. Therefore, frontalis sling surgery using a silicone rod could be a good treatment option in managing severe ptosis due to $3 \mathrm{rdNP}$ in cases showing the possibility of postoperative corneal complications.

To date, there are a few articles [6-8] reporting on frontalis sling surgery using a silicone rod related to $3 \mathrm{rdNP}$. Bagheri et al. [6] reported 18 patients of 3rdNP who received levator resection and frontalis sling procedures; however, the types of suspension materials used are mixed in sling surgery: silicone rods and Supramid (S. Jackson, Alexandria, VA, USA) or fascia. One case report [7] mentioned the efficacy of using silicone rods as a suspension material in frontalis sling surgery. However, in these papers, postoperative corneal status was not the main outcome measure. Until now, many studies [8-11] have covered the functional and cosmetic outcomes in frontalis sling surgery. However, in patients with 3rdNP who have poor corneal protective mechanism, the safety outcomes for the cornea should be clearly emphasized. This study aimed to investigate the surgical results of frontalis sling surgery using a silicone rod in 3rdNP by specifically focusing on the condition of the cornea. In addition, we investigated the safe eyelid height for the cornea postoperatively.

\section{Materials and Methods}

This retrospective, interventional case series was approved by the institutional review board of Seoul National University Bundang Hospital (No. B-2004-608-102). The current study involving the surgical procedure was carried out in adherence to the tenets of the Declaration of Helsinki. Informed consent was not required; the institutional review board granted a waiver of informed consent for this retrospective chart review study. All operations were performed by a single surgeon.

Patients with 3rdNP who underwent frontalis sling surgery with a silicone rod for ptosis correction between 2008 and 2019 were included in this study. The patients' clinical characteristics and surgical outcomes were retrospectively analyzed by reviews of medical records and photographs. The obtained data included information on sex, age, visual acuity, laterality of 3rdNP, extraocular movement, Bell's
A

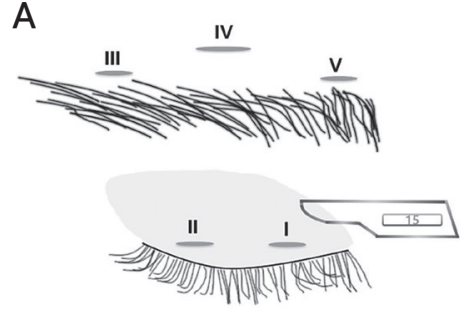

D

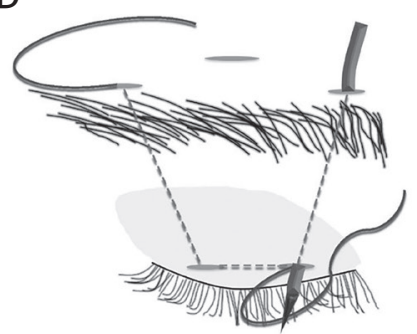

B

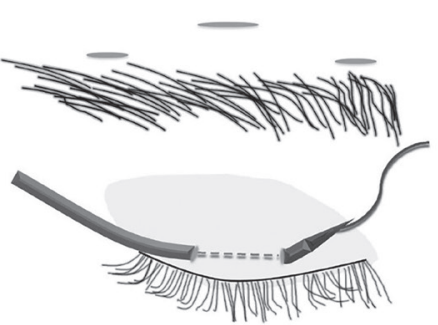

E

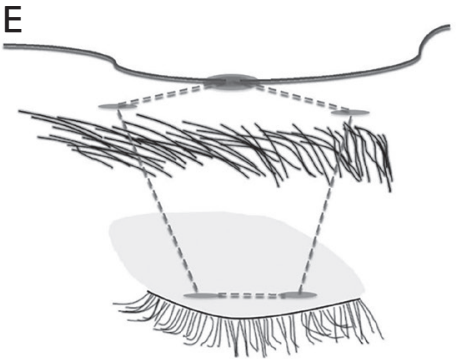

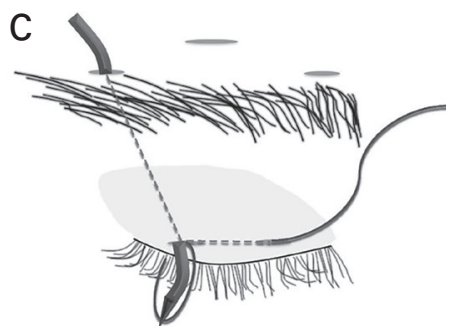

$\mathrm{F}$

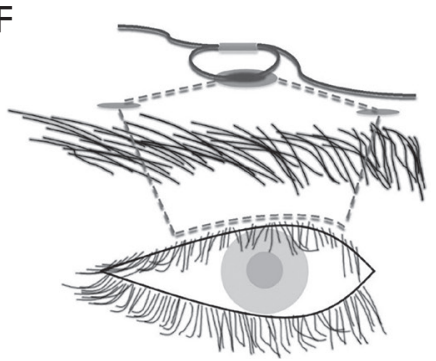

Fig. 1. Description of the sling surgery, drawn by the authors of this article. (A) Indication of five stab incision. (B) Passage of the rod through the two eyelid incision sites, from point I to point II. (C) Passage of the lateral end of the rod, from eyelid incision site (point II) to lateral suprabrow incision site (point III). (D) Passage of the medial end of the rod, from medial eyelid incision site (point I) to medial suprabrow incision site (point V). (E) Exit of both tips of the rod through the central suprabrow incision site (point IV). (F) Tips of the rod brought within a sleeve. 
phenomenon, levator function, preoperative and postoperative margin reflex distance (MRD), laterality of surgery, type of surgery performed, type of suspension material used, postsurgical status of the cornea, and presence of sling adjustment. The levator function was classified into three groups: poor (4 $\mathrm{mm}$ or less), moderate (between 5 and $7 \mathrm{~mm}$ ), and good ( $8 \mathrm{~mm}$ or more). The Bell's phenomenon was categorized into three stages: poor, moderate, and good.

\section{Surgical techniques}

Surgery was performed under local or general anesthesia depending on the patient's age. Lidocaine (2\%) mixed with 1:100 000 epinephrine was injected in the operation site. Five horizontal incisions were made with a No. 15 blade in a pentagonal shape, of which two were made $3 \mathrm{~mm}$ above the upper eyelid margin and three above the brow. Two horizontal incisions were made at the upper eyelid margin, and dissection was performed with sharp Wescott scissors through the orbicularis oculi muscle. After the plane of tarsus was exposed, two 6-0 polypropylene sutures were preplaced at the tarsal plate. After that, the Wright needle was passed above the tarsus, and the silicone rod (Visitec; BD Ophthalmics, Franklin Lakes, NJ, USA) was brought under the pretarsal orbicularis oculi. The silicone rod was fixed to the tarsal plate by tying the preplaced polypropylene sutures. A Jaeger lid plate was placed under the eyelid, and its tip was placed just under the superior orbital rim. The medial and lateral aspects of the silicone rod were passed towards the medial and lateral brow incisions, respectively, and then redirected toward the central brow in-

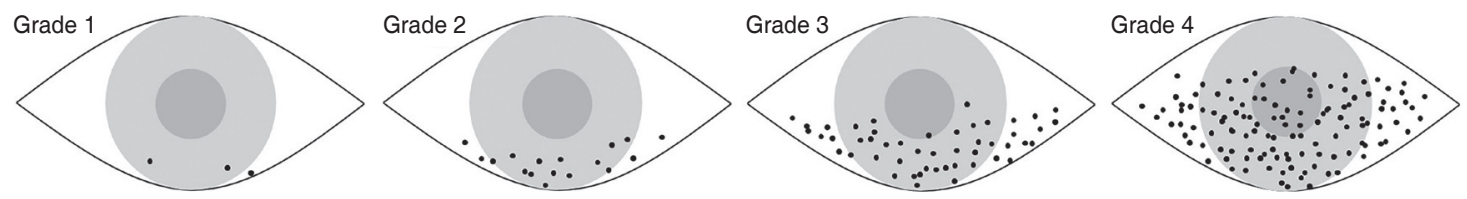

Fig. 2. Schema of postoperative corneal grading according to severity of erosions, drawn by the authors of this article.

Table 1. Demographic characteristics of the 18 patients in this study

\begin{tabular}{|c|c|c|c|c|c|}
\hline Patient no. & Sex & Age (yr) & Etiology of third cranial nerve palsy & Laterality & Follow-up period (mon) \\
\hline 1 & Male & 20 & Traumatic & Bilateral & 56 \\
\hline 2 & Male & 17 & Traumatic & Right & 3 \\
\hline 3 & Female & 64 & Traumatic & Right & 9 \\
\hline 4 & Male & 55 & Traumatic & Left & 19 \\
\hline 5 & Male & 31 & Congenital & Right & 11 \\
\hline 6 & Male & 5 & Inflammatory & Left & 4 \\
\hline 7 & Male & 50 & Vascular & Left & 45 \\
\hline 8 & Female & 26 & Tumor & Left & 63 \\
\hline 9 & Male & 59 & Vascular & Bilateral & 30 \\
\hline 10 & Female & 32 & Undetermined & Left & 87 \\
\hline 11 & Male & 42 & Vascular & Bilateral & 16 \\
\hline 12 & Male & 62 & Vascular & Left & 72 \\
\hline 13 & Male & 7 & Congenital & Bilateral & 20 \\
\hline 14 & Female & 54 & Congenital & Bilateral & 44 \\
\hline 15 & Female & 27 & Traumatic & Right & 43 \\
\hline 16 & Male & 8 & Congenital & Bilateral & 25 \\
\hline 17 & Female & 47 & Traumatic & Right & 2 \\
\hline 18 & Male & 26 & Congenital & Bilateral & 29 \\
\hline
\end{tabular}


cision with the Wright needle. The tips of the silicone rods were gathered together with a sleeve, and the upper eyelid was pulled up high until the MRD was $+1.0 \mathrm{~mm}$. The sleeve was tied with 6-0 polypropylene sutures and situated in the central brow incision. The subcutaneous tissue of the central incision was sutured with 6-0 vicryl. The skin incisions of the brow were closed with 6-0 polypropylene (Fig. 1A-1F).

Both, artificial tear eye drops and ointments, were started immediately after the frontalis sling operation. Patients visited our hospital at 1 week, 1 month, and 6 months postoperatively. Postoperative eyelid contour, eyelid height by MRD, and corneal condition were examined at each checkup. In cases showing overcorrected or undercorrected ptosis at 1 week, eyelid height was adjusted to achieve the targeted eyelid height.

\section{Corneal status evaluation}

The postoperative corneal status was categorized and evaluated as follows: grade 1, no or minimal erosions; grade 2 , mild erosions easily controlled by topical eye drops and ointments; grade 3, moderate erosions but controlled by medical therapy; and grade 4 , severe, persistent erosions or infiltration requiring additional surgery (Fig. 2).

Table 2. Preoperative and postoperative conditions of the 24 eyes of 18 patients in this study

\begin{tabular}{|c|c|c|c|c|c|c|}
\hline Patient no. & Bell's phenomenon & Preop MRD (mm) & Preop LF (mm) & $\begin{array}{l}\text { Postop 1-mon } \\
\text { MRD (mm) }\end{array}$ & $\begin{array}{l}\text { Last FU MRD } \\
(\mathrm{mm})\end{array}$ & $\begin{array}{l}\text { Postop corneal } \\
\text { status }^{*}\end{array}$ \\
\hline 1 & Poor & -4.0 & 4 & -0.5 & +1.0 & Grade 1 \\
\hline 1 & Poor & -4.0 & 6 & +0.5 & +1.5 & Grade 1 \\
\hline 2 & Poor & -3.0 & 5 & 0 & 0 & Grade 2 \\
\hline 3 & Moderate & -2.0 & 5 & +1.0 & +1.0 & Grade 1 \\
\hline 4 & Moderate & -1.0 & 5 & +1.0 & +0.5 & Grade 1 \\
\hline 5 & Poor & -5.0 & 2 & +3.0 & +2.0 & Grade 3 \\
\hline 6 & Good & +1.0 & 3 & $\mathrm{NE}$ & +2.0 & Grade 1 \\
\hline 7 & Poor & -6.0 & 4 & +0.5 & +1.0 & Grade 1 \\
\hline 8 & Poor & +1.0 & 8 & +2.0 & +1.0 & Grade 3 \\
\hline 9 & Poor & -6.0 & 0 & +2.0 & +2.0 & Grade 1 \\
\hline 9 & Poor & -6.0 & 0 & +2.0 & +2.0 & Grade 1 \\
\hline 10 & Poor & 0 & 0 & $\mathrm{NE}$ & +0.5 & Grade 1 \\
\hline 11 & Poor & -3.0 & 2 & +1.5 & +3.0 & Grade 2 \\
\hline 12 & Poor & -2.0 & 7 & +1.0 & +1.0 & Grade 1 \\
\hline 13 & Poor & -3.0 & 1 & +1.0 & +1.0 & Grade 1 \\
\hline 13 & Poor & -3.0 & 1 & +2.0 & +2.0 & Grade 1 \\
\hline 14 & Poor & 0 & 0 & +0.5 & +1.0 & Grade 2 \\
\hline 14 & Poor & +1.0 & 5 & +1.0 & +2.0 & Grade 2 \\
\hline 15 & Poor & -2.0 & 1 & +2.0 & +2.0 & Grade 2 \\
\hline 16 & Poor & -1.0 & 0 & -1.0 & 0 & Grade 3 \\
\hline 16 & Poor & -1.0 & 0 & 0 & 0 & Grade 3 \\
\hline 17 & Poor & 0 & 3 & +1.0 & +1.0 & Grade 1 \\
\hline 18 & Poor & -6.0 & 0 & +0.5 & +1.0 & Grade 1 \\
\hline 18 & Poor & -6.0 & 0 & +0.5 & +1.0 & Grade 1 \\
\hline
\end{tabular}

Preop = preoperative; $\mathrm{MRD}=$ margin reflex distance; $\mathrm{LF}=$ levator function; Postop = postoperative; FU = follow-up; NE = not evaluated.

*Grade 1, no or minimal erosions; grade 2, mild erosions easily controlled; grade 3, moderate erosions but controlled; and grade 4, severe, persistent erosions, or infiltration requiring additional surgery. 


\section{Statistical analysis}

Statistical analysis was performed using IBM SPSS ver. 22.0 (IBM Corp., Armonk, NY, USA). The variables were analyzed using the Mann-Whitney $U$-test, Kruskal-Wallis test, and linear-by-linear association. Statistical significance was set at $p<0.05$.

\section{Results}

A total of 24 eyes of 18 subjects with 3rdNP (12 male and six female patients) were enrolled in this study. Eleven of them showed a unilateral presentation, while seven showed a bilateral presentation of 3rdNP. The etiologies of 3rdNP were traumatic in six patients, congenital in five, vascular in four, tumor in one, inflammatory in one, and undetermined in one case. Frontalis sling operation with a silicone rod was performed on 24 eyelids of 18 patients; one patient who had presented with bilateral ptosis underwent unilateral surgery. Eleven of the 18 patients underwent strabismus surgery at the same time. The mean age at the time of ptosis surgery was 35.1 years (range, 5-64 years). The mean follow-up period was 32.1 months (range, 2-87 months).

The preoperative Bell's phenomenon was poor in 21 eyes $(87.5 \%)$, moderate in two $(8.3 \%)$, and good in one eye (4.2\%). The preoperative levator function was poor in 17 eyes $(70.8 \%)$, moderate in six $(25.0 \%)$, and good in one eye (4.2\%). Mean preoperative MRD was $-2.5 \mathrm{~mm}$ (range, -6.0 to $+1.0 \mathrm{~mm}$ ) and the mean MRD at 1 week after the operation was $+1.2 \mathrm{~mm}$ (range, -0.5 to $+3.0 \mathrm{~mm}$ ). The mean
MRD at 1 month after sling surgery, 6 months after surgery, and at the last follow-up was $+1.0 \mathrm{~mm}$ (range, -1.0 to $+3.0 \mathrm{~mm}$ ), $+1.1 \mathrm{~mm}$ (range, -1.0 to $+2.0 \mathrm{~mm}$ ), and $+1.2 \mathrm{~mm}$ (range, 0 to $+3.0 \mathrm{~mm}$ ), respectively. The demographic characteristics and clinical data of all patients are presented in Tables 1 and 2 .

Almost all patients achieved satisfactory eyelid height and contour after the frontalis sling operation with a silicone rod (Fig. 3A, 3B). In assessments of postoperative corneal status, 15 eyes $(62.5 \%)$ were categorized as grade 1 , five (20.8\%) as grade 2, and four (16.7\%) as grade 3 (Table 3). The four eyes of three patients with grade 3 corneal status maintained their corneal condition through medical treatment fairly well but underwent a sling loosening surgery because of difficulty in visiting our clinic regularly. No patients showed grade 4 corneal condition. The MRDs of the four eyes with grade 3 corneal status were $+2.0,+1.0,0$,

Table 3. Safety outcome according to postoperative corneal status

\begin{tabular}{lcc}
\hline Corneal status & No. of eyes & Mean last FU MRD (mm) \\
\hline Grade 1 & $15(62.5 \%)$ & +1.2 \\
Grade 2 & $5(20.8 \%)$ & +1.6 \\
Grade 3 & $4(16.7 \%)$ & +0.8 \\
Grade 4 & $0(0 \%)$ & -
\end{tabular}

$p=0.332$ (Kruskal-Wallis test between postoperative MRD and corneal status).

$\mathrm{FU}=$ follow-up; $\mathrm{MRD}=$ margin reflex distance.

"Grade 1, no or minimal erosions; grade 2, mild erosions easily controlled; grade 3, moderate erosions but controlled; and grade 4 , severe, persistent erosions or infiltration requiring additional surgery.
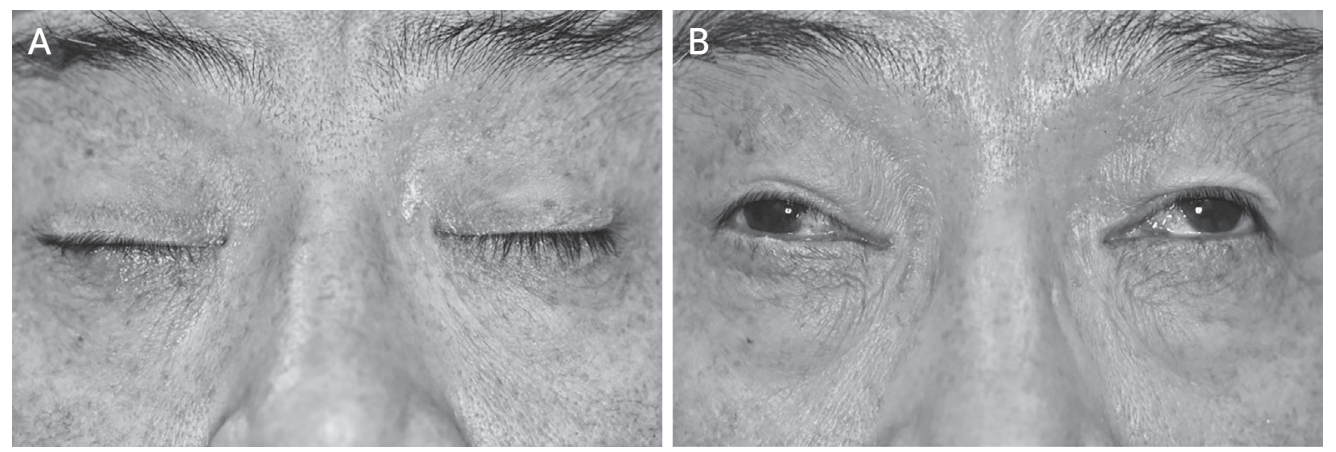

Fig. 3. A 59-year-old male patient with bilateral severe ptosis due to third nerve palsy. (A) Preoperative feature with a margin reflex distance of $-6 /-6$. (B) Twenty-nine months after bilateral sling surgery, with a margin reflex distance of $+2 /+2$. The patient was recommended for additional strabismus surgery. The patient provided informed consent for publication of the images. 
and $0 \mathrm{~mm}$ at the last follow-up. There was no significant association between postoperative MRD and corneal status (Kruskal-Wallis test, $p=0.332$ ). The degree of Bell's phenomenon and postoperative corneal status did not show a significant correlation either (linear-by-linear association, $p=0.228)$.

In a comparison of congenital and acquired 3rdNP, the mean preoperative MRD was not different between the two groups $(-2.7 \mathrm{~mm}$ in congenital $3 \mathrm{rdNP}$ and $-2.5 \mathrm{~mm}$ in acquired 3rdNP; Mann-Whitney $U$-test, $p=0.907$ ), and the degrees of Bell's phenomenon were not different either (linear-by-linear association, $p=0.189$ ). As for preoperative levator function, the congenital group showed poorer function than the acquired group $(1.0 \mathrm{~mm}$ in the congenital group and $3.5 \mathrm{~mm}$ in the acquired group, linear-by-linear association, $p=0.019$ ). However, surgical outcomes, including postoperative MRD and corneal status, were not different between the two groups (Mann-Whitney $U$-test, $p=0.726$; linear-by-linear association, $p=0.091$, respectively).

\section{Discussion}

We found that the frontalis sling operation using a silicone rod successfully treated ptosis with 3rdNP. Although poor Bell's phenomenon was very common, the postoperative corneal condition was good enough to be controlled medically.

Oculomotor palsy is a relatively common clinical diagnosis that mainly presents with diplopia and ptosis. 3rdNP can be congenital or acquired and the etiology of this disease is variable and complex. Many conditions have been reported to cause 3rdNP, such as trauma, aneurysms, pituitary tumors, cavernous sinus fistula, infarction, inflammation, infection, cavernous sinus thrombosis, and subarachnoid hemorrhage $[12,13]$. In our study, five patients were diagnosed with congenital 3rdNP and 13 with the acquired form. Despite the more severe ptosis (mean MRD, -2.7 vs. $-2.5 \mathrm{~mm}$ ) and poorer preoperative levator function $(1.0$ vs. $3.5 \mathrm{~mm})$ in congenital $3 \mathrm{rdNP}$, surgical outcomes in congenital 3rdNP were not significantly different from those in acquired 3rdNP in terms of eyelid height and corneal condition. This suggests that congenital 3rdNP can also be successfully managed with a frontalis sling operation with a silicone rod, even though ptosis was severe and levator function was poor in congenital 3rdNP cases.

The third cranial nerve originates from the nucleus in the midbrain and divides into the superior and inferior branches across the cavernous sinus before entering the orbit. The superior branch innervates the levator muscle and superior rectus muscle, and the inferior branch innervates the inferior rectus muscle, inferior oblique muscle, and medial rectus muscle $[14,15]$. 3rdNP can occur due to damage to any part of the pathway between the oculomotor nucleus in the midbrain and the extraocular muscles within the orbit $[12,16]$. Ptosis in the 3rdNP occurs due to damage to the superior branch of the third cranial nerve; hence, deterioration of superior rectus muscle function is frequently observed. In our study, all the patients showed various degrees of strabismus, and $15(83.3 \%)$ underwent strabismus surgery simultaneously or sequentially.

The method used for correcting ptosis depends on the patient's age, the cause and extent of the ptosis, the function of the levator muscle, the corneal perception state, and the presence or absence of Bell's phenomenon. Among these factors, the function of the levator muscle has the most important effect on the decision regarding surgical options [17]. The consensus for ptosis surgery recommends levator advancement when the levator function exceeds $10 \mathrm{~mm}$, levator resection for levator function of 6 to $10 \mathrm{~mm}$, and frontalis sling surgery for levator function of 4 to $5 \mathrm{~mm}$ or less $[18,19]$. Postsurgical corneal problems have been a major concern for ophthalmologists. Moreover, in patients with 3rdNP, the Bell's phenomenon is often poor due to eye movement limitations; hence, the decision to perform sling surgery is somewhat risky.

Fortunately, frontalis sling operations performed using a silicone rod can effectively raise the ptotic eyelid while preserving eyelid closure function [20]. Many doctors have reported favorable results for this suspension material. Carter et al. [4] used silicone rods in 61 eyes of 35 patients and achieved good eyelid height. Only nine eyes (15\%) with an absent or inadequate Bell's phenomenon showed postoperative exposure keratitis, which was well controlled medically. Bernardini et al. [21] performed surgery using silicone rods in 16 eyes of 10 patients and obtained satisfactory eyelid height. Only one patient (10\%) with absent Bell's phenomenon required additional surgery to manage corneal complications.

Various materials other than silicone have been used in frontalis sling surgery. Autologous fascia lata [22-24] has 
been used for over 100 years and remains one of the preferred materials. However, in young children, it is not easy to obtain autologous fascia lata; additional surgery is required on the leg, and scars may remain. To overcome these limitations, banked fascia lata has also been used. However, the recurrence rate with banked fascia lata is higher than that with autologous fascia lata, and there is a risk of infection [25]. Several synthetic materials such as polypropylene and nylon have been used for temporary suspension, but they may be associated with high recurrence rates [26]. In this study, we chose silicone rods as a suspension material in all patients for many reasons. First, the silicone rods exhibit excellent elasticity, allowing patients to close their upper eyelids with greater ease while maintaining good eyelid height [4]. Second, the silicone rods have superior adjustability. The rods are not incorporated into the surrounding tissues and allow easy adjustment of eyelid height after the surgery [5,27]. Third, the silicone rods have a long shelf life and are less susceptible to infection.

\section{Special emphasis on the cornea}

In many articles dealing with frontalis sling surgery [811], the primary outcome is the functional and cosmetic results involving eyelid contour, eyelid height, and symmetry. They also described possible postoperative complications, such as exposure keratopathy or lagophthalmos, suture granuloma, eyelid edema, entropion, wound infection, and so on. Among them, complications related to the cornea can be vision-threatening and very critical. Under normal conditions, the eye is well equipped with natural defense mechanisms to protect the ocular surface, especially the cornea [28]. The eyelids provide a physical barrier to dryness and trauma and prevent adherence of microorganisms to the ocular surface [29]. If this important barrier collapses due to incomplete eyelid closure after frontalis sling surgery, corneal erosion or severe exposure keratitis may occur. On the basis of this importance, we intended to assess the postoperative status of cornea as one of the main outcomes. The corneal condition after surgery, which means safety outcome, was classified into four grades. Twenty eyes out of $24(83.3 \%)$ showed minimal corneal erosions (grade I) or mild erosions (grade II) in our study. Considering the majority of our subjects had poor Bell's phenomenon, this result is fairly good. It can be hypothe- sized that because the probability of developing lagophthalmos is lowered due to the elasticity of silicone rods, corneal erosion is less likely to occur after surgery. A further comparative study with other sling materials is needed to verify this.

\section{Cosmesis versus safety}

The guidelines for desirable eyelid height after frontalis sling operation vary from study to study. Ho et al. [11] defined an excellent eyelid height as postoperative MRD of 3 $\mathrm{mm}$ or more. Dave et al. [30] reported that a favorable outcome was defined as MRD of $4 \mathrm{~mm}$ in bilateral cases. However, no studies have investigated the stability of the cornea according to the eyelid height. Postoperative eyelid cosmesis and corneal safety are like the ends of a seesaw; if the surgeon places too much emphasis on one aspect, it can lead to disappointing results in the other. Therefore, determining the ideal eyelid height to balance corneal safety and desirable cosmetic result is essential, especially in subjects with poor Bell's phenomenon. We thus sought to determine a safe upper limit of eyelid height for the postoperative cornea. However, the correlation between the postoperative MRD value and the postoperative corneal grade was not significant (Kruskal-Wallis test, $p=0.332$ ). This may imply that the corneal condition may be affected not only by MRD, but also by other factors such as tear film condition, elasticity of eyelids, and compliance with eye drops application. In other words, the corneal status may not be influenced by MRD alone. Although this study could not statistically reveal the safe eyelid height for the cornea, both cosmetic and safety outcomes were achieved in all subjects. We hope that our investigation will be a cornerstone for future ophthalmologic studies to find out the optimum eyelid height.

In conclusion, to the best of our knowledge, this is the largest series to report outcomes of frontalis sling surgery using a silicone rod in third nerve palsy. This study suggests a new method to define the postoperative safety outcome by specially focusing on categorized corneal status. Frontalis sling surgery using a silicone rod can effectively and safely correct ptosis in 3rdNP patients without severe corneal complications despite poor Bell's phenomenon. This finding can be helpful for surgical planning and counseling patients. The limitations of the current study include its retrospective nature, small sample size, and lack 
of a control group. Prospective large-scale studies are warranted to clarify the safety of frontalis sling surgery using a silicone rod in 3rdNP patients, and to find out the value of eyelid height safe for the cornea.

\section{Conflicts of Interest: None.}

Acknowledgements: None.

Funding: None.

\section{References}

1. Brazis PW. Isolated palsies of cranial nerves III, IV, and VI. Semin Neurol 2009;29:14-28.

2. Malone TJ, Nerad JA. The surgical treatment of blepharoptosis in oculomotor nerve palsy. Am J Ophthalmol 1988;105:57-64.

3. Crawford JS. Frontalis sling operation. J Pediatr Ophthalmol Strabismus 1982;19:253-5.

4. Carter SR, Meecham WJ, Seiff SR. Silicone frontalis slings for the correction of blepharoptosis: indications and efficacy. Ophthalmology 1996;103:623-30.

5. Ahn J, Kim NJ, Choung HK, et al. Frontalis sling operation using silicone rod for the correction of ptosis in chronic progressive external ophthalmoplegia. $\mathrm{Br} J$ Ophthalmol 2008;92:1685-8.

6. Bagheri A, Borhani M, Salehirad S, et al. Blepharoptosis associated with third cranial nerve palsy. Ophthalmic Plast Reconstr Surg 2015;31:357-60.

7. Bansal S, Das S. Frontalis sling surgery: a treatment modality for cyclic oculomotor nerve palsy. Saudi J Ophthalmol 2017;31:196-8.

8. Galindo-Ferreiro A, Akaishi P, Hanafi S, et al. Outcomes of two surgical techniques using silicone rod for frontalis sling suspension to treat severe ptosis. $J$ Pediatr Ophthalmol Strabismus 2017;54:52-8.

9. Lelli GJ Jr, Musch DC, Frueh BR, Nelson CC. Outcomes in silicone rod frontalis suspension surgery for high-risk noncongenital blepharoptosis. Ophthalmic Plast Reconstr Surg 2009;25:361-5.

10. Skaat A, Fabian D, Spierer A, et al. Congenital ptosis repair-surgical, cosmetic, and functional outcome: a report of 162 cases. Can J Ophthalmol 2013;48:93-8.

11. Ho YF, Wu SY, Tsai YJ. Factors associated with surgical outcomes in congenital ptosis: a 10-year study of 319 cases. Am J Ophthalmol 2017;175:173-82.
12. Raza HK, Chen H, Chansysouphanthong T, Cui G. The aetiologies of the unilateral oculomotor nerve palsy: a review of the literature. Somatosens Mot Res 2018;35:229-39.

13. Phuljhele S, Dhiman R, Sharma M, et al. Acquired ocular motor palsy: current demographic and etiological profile. Asia Pac J Ophthalmol (Phila) 2020;9:25-8.

14. Bruce BB, Biousse V, Newman NJ. Third nerve palsies. Semin Neurol 2007;27:257-68.

15. Nagendran ST, Lee V, Perry M. Traumatic orbital third nerve palsy. Br J Oral Maxillofac Surg 2019;57:578-81.

16. Nakagawa Y, Toda M, Shibao S, Yoshida K. Delayed and isolated oculomotor nerve palsy following minor head trauma. Surg Neurol Int 2017;8:20.

17. Mauriello JA, Wagner RS, Caputo AR, et al. Treatment of congenital ptosis by maximal levator resection. Ophthalmology 1986;93:466-9.

18. Berke RN. Results of resection of the levator muscle through a skin incision in congenital ptosis. AMA Arch Ophthalmol 1959;61:177-201.

19. Fasanella RM, Servat J. Levator resection for minimal ptosis: another simplified operation. Arch Ophthalmol 1961;65:493-6.

20. Bansal RK, Sharma S. Results and complications of silicone frontalis sling surgery for ptosis. J Pediatr Ophthalmol Strabismus 2015;52:93-7.

21. Bernardini FP, de Conciliis C, Devoto MH. Frontalis suspension sling using a silicone rod in patients affected by myogenic blepharoptosis. Orbit 2002;21:195-8.

22. Leibovitch I, Leibovitch L, Dray JP. Long-term results of frontalis suspension using autogenous fascia lata for congenital ptosis in children under 3 years of age. Am J Ophthalmol 2003;136:866-71.

23. Yoon JS, Lee SY. Long-term functional and cosmetic outcomes after frontalis suspension using autogenous fascia lata for pediatric congenital ptosis. Ophthalmology 2009;116:1405-14.

24. Philandrianos C, Galinier P, Salazard B, et al. Congenital ptosis: long-term outcome of frontalis suspension using autogenous temporal fascia or fascia lata in children. J Plast Reconstr Aesthet Surg 2010;63:782-6.

25. Wilson ME, Johnson RW. Congenital ptosis. Long-term results of treatment using lyophilized fascia lata for frontalis suspensions. Ophthalmology 1991;98:1234-7.

26. Wasserman BN, Sprunger DT, Helveston EM. Comparison of materials used in frontalis suspension. Arch Ophthalmol 2001;119:687-91. 
27. Wong VA, Beckingsale PS, Oley CA, Sullivan TJ. Management of myogenic ptosis. Ophthalmology 2002;109:1023-31.

28. McClellan KA. Mucosal defense of the outer eye. Surv Ophthalmol 1997;42:233-46.

29. Suresh P, Mercieca F, Morton A, Tullo AB. Eye care for the critically ill. Intensive Care Med 2000;26:162-6.

30. Dave TV, Sharma P, Nayak A, et al. Outcomes of frontalis sling versus levator resection in patients with monocular elevation deficiency associated ptosis. Ophthalmic Plast Reconstr Surg 2019;35:251-5. 